Journal of Thermal Engineering, Vol. 5, No. 1, pp. 76-92, January, 2019

Yildiz Technical University Press, Istanbul, Turkey

\title{
ANALYTICAL DECOMPOSITION SOLUTIONS FOR HEAT TRANSFER ON STRAIGHT FINS WITH TEMPERATURE DEPENDENT THERMAL CONDUCTIVITY AND INTERNAL HEAT GENERATION
}

\author{
Akinbowale T. Akinshilo ${ }^{1 *}$
}

\begin{abstract}
This paper analyses heat transfer across straight convecting fins with temperature dependent thermal conductivity and internal heat generation using the Adomian decomposition method (ADM). The ADM is the preferred analytical scheme adopted to provide approximate solutions to nonlinear equations arising from the dependence of thermal conductivity and heat transfer coefficient on temperature distribution. The effect of parameters such as internal heat generation, thermo geometric and thermal conductivity on the temperature profile and heat flux is studied. Where results reveal that thermo geometric parameter and thermal conductivity causes a significant increase in heat transfer across fin base. This study provides useful insight to fins operational performance in applications such as radiators, boilers, refrigeration devices, oil pipelines amongst others. Comparison of solutions with existing works in literature forms good agreement.
\end{abstract}

\section{Keywords: Fins, Temperature Dependent Viscosities, Convection, Heat Generation, Adomian Decomposition Method}

\section{INTRODUCTION}

Overtime fins have found useful applications in heat transfer equipments due to its relatively large area which enhances heat transfer. Therefore serving as a means of effective heat exchange medium in applications such as automobiles, steam power plants,boilers, air-conditioning and refrigeration devices amongst others. Many research effort have been expended over the years in the study, analysis and experimental investigations of the fins which is of vast importance in the field of science and engineering.

In efforts to study fins Atay and Coskun [1] presented a comparative analysis of fin efficiency using analytical and numerical techniques to better ascertain the accuracy of the analytical solutions while Chowdhury et al. [2] compared analytical solutions for non-linear fin problems applying the homotopy analysis method (HAM) and homotopy pertubation method (HPM) where they showed that the HAM proves to be more accurate. Kraus [3] et al. studied heat transfer in extended surfaces for the purpose of optimising heat transfer in large media. Ghasemi et al. [4] analysed convective fins with temperature dependent thermal conductivity and heat generation applying the differential transform method(DTM) where results confirms the accuracy and convieniency of the DTM . The efficiency of fully wet semi-spherical porous fins was studied by Hatami et al. [5] where the effect of porosity is investigated on fins efficiency shortly after numerical and experimental investigation of heat exchangers was presented by Hatami et al. [6-8] where they optimised the operating condition and were also able to recover waste heat from the heat exchangers. Analytical solutions was developed by Fernandez et al. [9] to improve thermal performance on fins using nonlinear models. In the bid to efficiently analyse straight fins Sadris et al. [10] studied convective heat transfer in fins with thermal conductivity and variable heat transfer. Differential transform method was applied to the energy equation of a thermal conductive straight fins with three profiles by Moradi and Almadikia [11] while Joneidi et al. [12] studied fin efficiency with temperature dependent thermal conductivity using the same method analyzing the effect of physical paramters on heat transfer. Thermal behaviour of longitudinal fins under convective and radiative heat transfer was studied by Hatami and Ganji [13] considering different shapes and ceramic materials where they proved Silicon nitride is a very good material as a result of its high amount of heat transfer shortly after they [14] extended their study to fully wet porous fins considering This paper was recommended for publication in revised form by Regional Editor Jaap Hoffman

${ }^{1}$ Department of Mechanical Engineering, University of Lagos, Akoka- Yaba, Lagos, Nigeria

${ }^{\star} E$-mail address: ta.akinshilo@gmail.com

Orcid id: $h$ ttps://orcid.org/0000-0002-6436-3420

Manuscript Received 15 June 2017, Accepted 08 August 2017 
temperature and humidity differences as driving forces of heat and mass transfer. Approximate analytical solution was utilised by Arslanturk [15] to investigate efficiency of fins where fins temperature distribution was determined. Aziz and Enamul [16] presented analytical solutions developed by pertubations scheme for straight convecting fins to study its thermal performance.Shortly after Aziz [17] investigated convecting fins with internal heat generation still adopting the pertubation solutions.

In recent times, the study of fins with temperature dependent thermal conductivity has proven an exciting area to researchers [18-27] due to fins having large temperature difference between the base and tip.Otherwise thermal conductivity is assumed constant which is the case in ordinary fins. Temperature equation governing the heat transfer problem becomes nonlinear due to its dependence on coefficient of heat transfer and thermal conductivity which makes the exact solutions difficult to obtain. Therefore the use of numerical and analytical approximate solutions was applied by researchers [1-27]. Methods of solutions utilised include the pertubation method (PM),homotopy analysis method (HAM), homotopy pertubation method (HPM), variational iteration method (VIM) and garlerkin method of weighted residuals. Methods such as PM are limited owing to the problems of weak nonlinearities and artificial pertubation parameter. The need to find a initial condition to satisfy the boundary condition makes methods such as HPM,VIM,DTM,HAM requires computational tools in handling a solution of large parameters resulting to large computational cost and time [28-35]. The garlerkin's finite element of weighted residual, no doubt a powerful analytical method requires the weighted residuals to satisfy weighting functions which may be arbitrary [27]. The method of solutions of decomposing nonlinear coupled equations into linear and nonlinear terms makes the Adomian Decomposition Method (ADM) a powerful,yet relatively simplistic method which is not limited by any artificial parameter or initial guess term. These makes ADM an interesting scheme in providing analytical solutions to nonlinear problems in science and engineering as often employed by researchers .

Motivated by past works this paper aim to investigate heat transfer in convective straight fins with thermal dependent conductivity and internal heat generation. Adomian decomposition method is adopted in analyzing the coupled nonlinear equation which is used to investigate temperature distribution, heat flux at fins base and effiency.

\section{MODEL DEVELOPMENT AND ANALYTICAL SOLUTIONS}

A straight fin with constant heat transfer and temperature is considered as described in the model diagram figure 1 . The fin thermal conductivity and internal heat generation are temperature dependent, both sides of the fins are exposed to ambient environmental condition. It is assumed that the fin base as a uniform temperature, surrounding media temperature is uniform,tip of the fin is insulated, there's no contact resistance between fin base and prime surface and heat transfer from fin edges and temperature gradient across fin thickness is negligible. Therefore going by the above assumptions the governing equation can be reduced to the following pair of ordinary differential equation which is introduced as :

$$
\frac{d}{d x}\left[K(T) \frac{d T}{d x}\right]-\frac{h}{A_{c}} P\left(T-T_{\infty}\right)-q(T)=0
$$

with boundary considition considered as,

$$
\begin{array}{ll}
x=0 & \frac{d T}{d x}=0 \\
x=L & T=T_{b}
\end{array}
$$


Journal of Thermal Engineering, Research Article, Vol. 5, No. 1, pp. 76-92, January, 2019

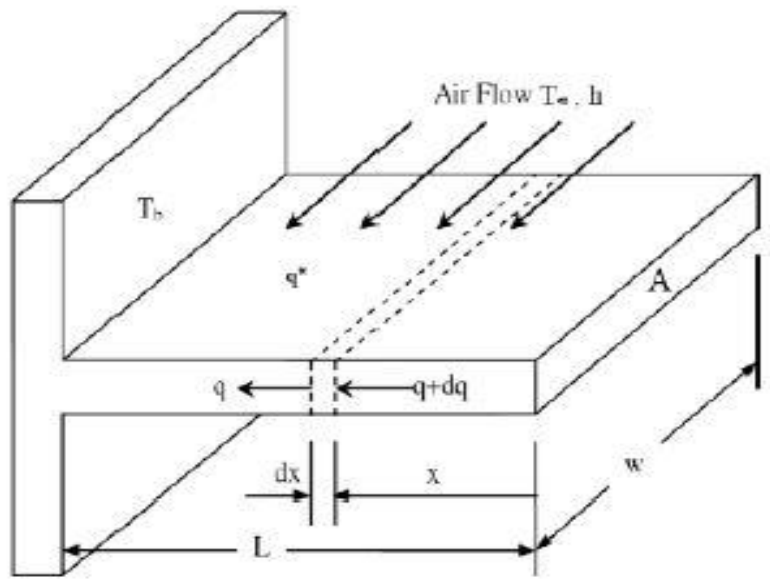

Figure 1. Physical model of problem [4]

Temperature dependent thermal properties is given by,

$$
K(T)=K_{a}\left[1+\lambda\left(T-T_{\infty}\right)\right]
$$

Internal heat generation is given by

$$
q(T)=q_{a}\left[1+\psi\left(\mathrm{T}-\mathrm{T}_{\infty}\right)\right]
$$

Substituting equations (3) and (4) into (1) yields

$$
\frac{d}{d x}\left[K_{a}\left[1+\lambda\left(T-T_{\infty}\right)\right] \frac{d T}{d x}\right]-\frac{h_{b} P\left(T-T_{\infty}\right)}{A_{C}}+q_{a}\left[1+\psi\left(T-T_{\infty}\right)\right]=0
$$

with the dimensionless parameters stated in the nomenclature equation (5) can be expressed as [4]:

$$
\frac{d^{2} \theta}{d x^{2}}+\beta \theta \frac{d^{2} \theta}{d x^{2}}+\beta\left(\frac{d \theta}{d x}\right)^{2}-M^{2} \theta+M^{2} Q+M^{2} Q \gamma \theta=0
$$

The appropriate boundary condition is given as 


$$
\begin{array}{ll}
x=0 & \frac{d \theta}{d x}=0 \\
x=1 & \theta=1
\end{array}
$$

\section{ADOMIAN DECOMPOSITION METHOD PRINCIPLES}

The adomian decomposition method is used for providing approximate or semi approximate analytical solutions to a wide range of problems involving integral,differential and algebraic equations. Here the non-linear equation is described as,

$$
L u+R u+N u=g
$$

where $\mathrm{L}$ is the highest order, $\mathrm{R}$ is the linear term, $\mathrm{N}$ is the nonlinear term and $\mathrm{g}$ is the source term. Applying an inverse operator $\mathrm{L}^{-1}$ yields,

$$
u=f(x)-L^{-1}(R u)-L^{-1}(N u)
$$

where $f(x)$ is the term obtained from integrating the source term. The non linear operator is expressed as an infinite series referred to as the Adomian polynomials, $N u=F(u)$

$$
F(u)=\sum_{n=0}^{\infty} A_{n}
$$

By performing the series expansion of $A_{n}$. Higher order Polynomials are generated.The adomian method defines the solution $\mathrm{u}(\mathrm{x})$ by the series

$$
u=\sum_{n=0}^{\infty} u_{n}
$$

The series expansion of $F(u)$ is an infinite series, which is expressed using the Taylor series as,

$$
F(u)=\mathrm{F}\left(\mathrm{u}_{0}\right)+\frac{d F}{d X}\left(\mathrm{u}_{0}\right)\left(\mathrm{u}-\mathrm{u}_{0}\right)+\frac{d^{2} F}{d X^{2}}\left(u_{0}\right) \frac{\left(u-u_{0}\right)^{2}}{2 !}+\frac{d^{3} F}{d X^{3}}\left(u_{0}\right) \frac{\left(u-u_{0}\right)^{3}}{3 !}+\ldots \ldots
$$

The above is rewritten as $u-u_{0}=u_{1}+u_{2}+u_{3}+\ldots \ldots$, substituting equation (12) then equating the expressions for $F(u)$ in equation (10) defines the formulae for the Adomian polynomial as [15]:

$$
F(u)=A_{1}+A_{2}+\ldots .=F\left(u_{0}\right)+\frac{d F}{d X}\left(u_{0}\right)\left(\mathrm{u}_{1}+u_{2}+\ldots\right)+\frac{d^{2} F}{d X^{2}}\left(u_{0}\right) \frac{\left(u_{1}+u_{2}+\ldots . .\right)^{2}}{2 !}+\ldots . .
$$


Journal of Thermal Engineering, Research Article, Vol. 5, No. 1, pp. 76-92, January, 2019

\section{APPLICATION OF ADM}

The adomian decomposition method (ADM) is adopted in generating solutions to the coupled ordinary nonlinear second order differential equation which may be expressed as:

$$
L_{x x}(\theta)=-\beta \theta \frac{d^{2} \theta}{d x^{2}}-\beta\left(\frac{d \theta}{d x}\right)^{2}-M^{2} \theta+M^{2} Q-M^{2} Q \gamma \theta
$$

In order to escape difficult integrations the highest order differential operator was taken as $L_{x x}=\frac{d^{2}}{d x^{2}}$ for the coupled equation.Thus inverting $L_{x x}$ gives $L_{x x}^{-1}$. On applying $L_{x x}^{-1}$ to equation (8) gives,

$$
\theta=C_{1}+C_{2} x+L_{x x}^{-1}\left[-\beta \theta \frac{d^{2} \theta}{d x^{2}}-\beta\left(\frac{d \theta}{d x}\right)^{2}-M^{2} \theta-M^{2} Q \gamma \theta\right]+L_{x x}^{-1} M^{2} Q
$$

with respect to ADM, $\theta$ may be expressed in the form,

$$
\theta=\sum_{n=0}^{\infty} \theta_{n}
$$

Utilising equations (16) in (15) yields,

$$
\theta=C_{1}+C_{2} x+L_{x x}^{-1}\left(M^{2} Q\right)+L_{x x}^{-1}\left[\left(-\beta \sum_{n=0}^{\infty} \theta_{n} \frac{d^{2}}{d x^{2}} \sum_{n=0}^{\infty} \theta_{n}\right)-\left(\beta \frac{d}{d x} \sum_{n=0}^{\infty}\left(\theta_{n}\right)^{2}\right)+\left(M^{2} \sum_{n=0}^{\infty} \theta_{n}\right)-M^{2} Q \gamma \sum_{n=0}^{\infty} \theta_{n}\right]
$$

The nonlinear terms will be explored in the form of $\Lambda_{\mathrm{n}}$ in the Adomian polynomials which yields,

$$
\sum_{n=0}^{\infty} \Lambda_{n}=\left[\left(-\beta \sum_{n=0}^{\infty} \theta_{n} \frac{d^{2}}{d x^{2}} \sum_{n=0}^{\infty} \theta_{n}\right)-\left(\beta \frac{d}{d x} \sum_{n=0}^{\infty}\left(\theta_{n}\right)^{2}\right)+\left(M^{2} \sum_{n=0}^{\infty} \theta_{n}\right)-M^{2} Q \gamma \sum_{n=0}^{\infty} \theta_{n}\right]
$$

Utilising equation (18), the equation (17) may be expressed as, 
Journal of Thermal Engineering, Research Article, Vol. 5, No. 1, pp. 76-92, January, 2019

$$
\theta=C_{1}+C_{2} x+L_{x x}^{-1}\left(M^{2} Q\right)+L_{x x}^{-1}\left(\sum_{n=0}^{\infty} \Lambda_{n}\right)
$$

where boundary conditions takes the form,

$$
\begin{aligned}
& \mathrm{x}=0 \quad \sum_{n=0}^{\infty} \theta_{n}=0 \\
& \mathrm{x}=1 \quad \sum_{n=0}^{\infty} \theta_{n}=1
\end{aligned}
$$

The zeroth order can be obtained from the recursive relations equations (19) and (20),

$$
\theta_{0}=C_{1}+C_{2} x-L_{x x}^{-1} M^{2} Q
$$

with leading order boundary condition expressed as,

$$
\begin{array}{ll}
x=0 & \frac{d \theta_{0}}{d x}=0 \\
x=1 & \theta_{0}=1
\end{array}
$$

The remaining order of the solutions is given as,

$$
u_{j+1}=L_{x x}^{-1}\left(\Lambda_{j}\right), \quad j \geq 0
$$

with boundary condition expressed as,

$$
\begin{aligned}
& \sum_{n=0}^{\infty} \theta_{n}=0 \text { at } \mathrm{x}=0 \\
& \sum_{n=0}^{\infty} \theta_{n}=1 \text { at } \mathrm{x}=1
\end{aligned}
$$

From equation (18) the Adomian polynomians $\Lambda$ can be obtained as,

$$
\Lambda_{0}=M^{2} \theta_{0}-\beta \theta_{0} \frac{d^{2} \theta_{0}}{d x^{2}}-\beta\left(\frac{d \theta_{0}}{d x}\right)^{2}-M^{2} Q \gamma \theta_{0}
$$


Journal of Thermal Engineering, Research Article, Vol. 5, No. 1, pp. 76-92, January, 2019

$$
\Lambda_{1}=M^{2} \theta_{1}-\beta \theta_{0} \frac{d^{2} \theta_{1}}{d x^{2}}-\beta \theta_{1} \frac{d^{2} \theta_{0}}{d x^{2}}-2 \beta \frac{d \theta_{0}}{d x} \frac{d \theta_{1}}{d x}-M^{2} Q \gamma \theta_{1}
$$

Zeroth order solution can be obtained by simplifying the recursive relation equation (21) using the leading order boundary condition equation (22) which yields.

$$
\theta_{0}=1-\frac{M^{2} Q}{2}\left(1-x^{2}\right)
$$

First order solution can be obtained from equation (23) and (24) which is expressed as,

$$
\theta_{1}=L_{x x}^{-1}\left(\Lambda_{0}\right)
$$

with the first order boundary condition as follows,

$$
\begin{aligned}
& x=0 \quad \frac{d \theta_{1}}{d x}=0 \\
& x=1 \quad \theta_{1}=1
\end{aligned}
$$

Substituting equation (25) into (28) . Upon simplifying with the aid of first order boundary condition equation (29) can be easily shown as,

$$
\begin{aligned}
& \theta_{1}=-\frac{M^{2}}{2}\left(1-x^{2}\right)+\frac{M^{4} Q}{24}\left(5-6 x^{2}+x^{4}\right)+\frac{\beta M^{2} Q}{2}\left(1-x^{2}\right)-\frac{\beta M^{4} Q^{2}}{24}\left(5-6 x^{2}+x^{4}\right)+\frac{\beta M^{4} Q^{2}}{30}\left(1-x^{6}\right) \\
& +\frac{M^{2} Q \gamma}{2}\left(1-x^{2}\right)-\frac{M^{4} Q^{2} \gamma}{24}\left(5-6 x^{2}+x^{4}\right)
\end{aligned}
$$

Second order solution can be obtained from equation (23) and (24) which is expressed as,

$$
\begin{aligned}
& \theta_{2}=L_{x x}^{-1}\left(\Lambda_{1}\right) \\
& x=0 \quad \frac{d \theta_{2}}{d x}=0 \\
& x=1 \quad \theta_{2}=1
\end{aligned}
$$

Substituting equation (26) into (31). Upon simplifying with the aid of second order boundary condition equation (32) can be easily shown as, 
Journal of Thermal Engineering, Research Article, Vol. 5, No. 1, pp. 76-92, January, 2019

$$
\begin{aligned}
& \theta_{2}=\left[M^{2}-M^{2} Q \gamma-\beta M Q\right]\left[\frac{M^{2}}{24}\left(5-6 x^{2}+x^{4}\right)-\frac{M^{4} Q}{720}\left(61-75 x^{2}+15 x^{4}-x^{6}\right)-\right. \\
& \frac{\beta M Q}{24}\left(5-6 x^{2}+x^{4}\right)+\frac{\beta M^{4} Q^{2}}{720}\left(61-75 x^{2}+15 x^{4}-x^{6}\right)-\frac{\beta M^{4} Q^{2}}{1680}\left(27-28 x^{2}+x^{8}\right)- \\
& \left.\frac{M^{2} Q \gamma}{24}\left(5-6 x^{2}+x^{4}\right)+\frac{M^{4} Q^{2} \gamma}{720}\left(61-75 x^{2}+15 x^{4}-x^{6}\right)\right]+\frac{\beta M^{2}}{2}\left(1-x^{6}\right)+\frac{\beta M^{4} Q}{24}\left(5+6 x^{2}-x^{4}\right) \\
& -\frac{\beta^{2} M^{2} Q}{2}\left(1-x^{2}\right)-\frac{\beta^{2} M^{4} Q^{2}}{24}\left(5+6 x^{2}-x^{4}\right)-\frac{\beta^{2} M^{4} Q^{2}}{30}\left(1-x^{6}\right)-\frac{\beta M^{2} Q \gamma}{2}\left(1-x^{2}\right)- \\
& \frac{\beta M^{4} Q^{2} \gamma}{24}\left(5+6 x^{2}-x^{4}\right)+\frac{\beta M^{6} Q^{2}}{120}\left(11+15 x^{2}-5 x^{4}-x^{6}\right)-\frac{\beta^{2} M^{4} Q^{2}}{24}\left(5+6 x^{2}-x^{4}\right)- \\
& \frac{\beta^{2} M^{6} Q^{3}}{120}\left(11-15 x^{2}+5 x^{4}-x^{6}\right)+\frac{\beta^{2} M^{6} Q^{3}}{1680}\left(13-28 x^{6}+15 x^{8}\right)+\frac{\beta M^{4} Q^{2} \gamma}{24}\left(5-6 x^{2}+x^{4}\right) \\
& -\frac{\beta M^{6} Q^{3} \gamma}{120}\left(11-15 x^{2}+5 x^{4}-x^{6}\right)+\frac{\beta M^{4} Q}{6}\left(1-x^{4}\right)-\frac{\beta M^{6} Q^{2}}{180}\left(13-15 x^{4}+2 x^{6}\right)- \\
& \frac{\beta^{2} M^{4} Q^{2}}{6}\left(1-x^{4}\right)+\frac{\beta^{2} M^{6} Q^{3}}{360}\left(13-15 x^{4}+2 x^{6}\right)-\frac{\beta^{2} M^{6} Q^{3}}{140}\left(1-x^{8}\right)-\frac{\beta M^{4} Q^{2} \gamma}{2}\left(1-x^{4}\right)+ \\
& \frac{\beta M^{6} Q^{3} \gamma}{180}\left(13-15 x^{4}+2 x^{6}\right)
\end{aligned}
$$

The summations of equations (27),(30) and (33) gives the ADM solutions for the temperature profile which is expressed as

$$
\begin{aligned}
& \theta=1-\frac{M^{2} Q}{2}\left(1-x^{2}\right)-\frac{M^{2}}{2}\left(1-x^{2}\right)+\frac{M^{4} Q}{24}\left(5-6 x^{2}+x^{4}\right)+\frac{\beta M^{2} Q}{2}\left(1-x^{2}\right)- \\
& \frac{\beta M^{4} Q^{2}}{24}\left(5-6 x^{2}+x^{4}\right)+\frac{\beta M^{4} Q^{2}}{30}\left(1-x^{6}\right)+\frac{M^{2} Q \gamma}{2}\left(1-x^{2}\right)-\frac{M^{4} Q^{2} \gamma}{24}\left(5-6 x^{2}+x^{4}\right)+ \\
& {\left[M^{2}-M^{2} Q \gamma-\beta M Q\right]\left[\frac{M^{2}}{24}\left(5-6 x^{2}+x^{4}\right)-\frac{M^{4} Q}{720}\left(61-75 x^{2}+15 x^{4}-x^{6}\right)-\right.} \\
& \frac{\beta M Q}{24}\left(5-6 x^{2}+x^{4}\right)+\frac{\beta M^{4} Q^{2}}{720}\left(61-75 x^{2}+15 x^{4}-x^{6}\right)-\frac{\beta M^{4} Q^{2}}{1680}\left(27-28 x^{2}+x^{8}\right)- \\
& \left.\frac{M^{2} Q \gamma}{24}\left(5-6 x^{2}+x^{4}\right)+\frac{M^{4} Q^{2} \gamma}{720}\left(61-75 x^{2}+15 x^{4}-x^{6}\right)\right]+\frac{\beta M^{2}}{2}\left(1-x^{6}\right)+\frac{\beta M^{4} Q}{24}\left(5+6 x^{2}-x^{4}\right) \\
& -\frac{\beta^{2} M^{2} Q}{2}\left(1-x^{2}\right)-\frac{\beta^{2} M^{4} Q^{2}}{24}\left(5+6 x^{2}-x^{4}\right)-\frac{\beta^{2} M^{4} Q^{2}}{30}\left(1-x^{6}\right)-\frac{\beta M^{2} Q \gamma}{2}\left(1-x^{2}\right)- \\
& \frac{\beta M^{4} Q^{2} \gamma}{24}\left(5+6 x^{2}-x^{4}\right)+\frac{\beta M^{6} Q^{2}}{120}\left(11+15 x^{2}-5 x^{4}-x^{6}\right)-\frac{\beta^{2} M^{4} Q^{2}}{24}\left(5+6 x^{2}-x^{4}\right)- \\
& \frac{\beta^{2} M^{6} Q^{3}}{120}\left(11-15 x^{2}+5 x^{4}-x^{6}\right)+\frac{\beta^{2} M^{6} Q^{3}}{1680}\left(13-28 x^{6}+15 x^{8}\right)+\frac{\beta M^{4} Q^{2} \gamma}{24}\left(5-6 x^{2}+x^{4}\right) \\
& -\frac{\beta M^{6} Q^{3} \gamma}{120}\left(11-15 x^{2}+5 x^{4}-x^{6}\right)+\frac{\beta M^{4} Q}{6}\left(1-x^{4}\right)-\frac{\beta M^{6} Q^{2}}{180}\left(13-15 x^{4}+2 x^{6}\right)- \\
& \frac{\beta^{2} M^{4} Q^{2}}{6}\left(1-x^{4}\right)+\frac{\beta^{2} M^{6} Q^{3}}{360}\left(13-15 x^{4}+2 x^{6}\right)-\frac{\beta^{2} M^{6} Q^{3}}{140}\left(1-x^{8}\right)-\frac{\beta M^{4} Q^{2} \gamma}{2}\left(1-x^{4}\right)+ \\
& \frac{\beta M^{6} Q^{3} \gamma}{180}\left(13-15 x^{4}+2 x^{6}\right)
\end{aligned}
$$

Conductive heat transfer at the base of the fin is obtained from the generalised fourier conduction heat equation which is expressed as 
Journal of Thermal Engineering, Research Article, Vol. 5, No. 1, pp. 76-92, January, 2019

$$
q_{b}=A_{c} k(T) \frac{d T}{d x}
$$

Non-dimensionalizing equation (35), heat transfer rate at fins base is defined as [27]

$$
\overline{q_{b}}=(1+\beta \theta) \frac{d \theta}{d x}
$$

Upon substituting parameters from the analysed temperature profile equation (34) in (36). The heat transfer at the fin base may simply be shown as

The ratio of actual heat transfer from fins surface to heat transfer from the surface if the entire fin is at the same temperature as the base is regarded as the fins efficiency which is derived as [27]:

$$
\eta=\frac{Q}{Q_{\text {ideal }}}=\frac{\int_{0}^{b} \mathrm{P}\left(\mathrm{T}-\mathrm{T}_{a}\right) d x}{P b\left(T_{b}-T_{a}\right)}=\int_{0}^{1} \theta(x) d x
$$

Therefore fins efficiency can be obtained upon simplifying the equation 38 which can be easily shown as,

$$
\begin{aligned}
& \eta=M^{2} Q+M^{2} Q-\frac{M^{4} Q}{3}-\beta M^{2} Q+\frac{\beta M^{4} Q^{2}}{3}-\frac{\beta M^{4} Q^{2}}{5}-M^{2} Q \gamma+\frac{M^{4} Q^{2} \gamma}{3}+\left[M^{2}-M^{2} Q \gamma+\beta M Q\right]^{*} \\
& {\left[-\frac{M^{2}}{3}+\frac{96 M^{4} Q}{720}+\frac{\beta M Q}{3}-\frac{96 \beta M^{4} Q^{2}}{720}+\frac{49 \beta M^{4} Q^{2}}{1680}+\frac{M^{2} Q \gamma}{3}-\frac{96 M^{4} Q^{2} \gamma}{720}\right]-3 \beta M^{2}+\frac{\beta M^{4} Q}{3}+\beta^{2} M^{2} Q} \\
& -\frac{\beta^{2} M^{4} Q^{2}}{3}+\frac{\beta^{2} M^{4} Q^{2}}{5}+\beta M^{2} Q \gamma-\frac{\beta M^{4} Q^{2} \gamma}{3}+\frac{\beta M^{6} Q^{2}}{30}-\frac{\beta^{2} M^{4} Q^{2}}{3}+\frac{2 \beta^{2} M^{6} Q^{3}}{15}-\frac{\beta^{2} M^{6} Q^{3}}{35}- \\
& \frac{\beta M^{4} Q^{2} \gamma}{3}+\frac{16 \beta M^{6} Q^{3} \gamma}{120}-\frac{2 \beta M^{4} Q}{3}+\frac{48 \beta M^{6} Q^{2}}{120}+\frac{2 \beta^{2} M^{4} Q^{2}}{3}-\frac{48 \beta^{2} M^{6} Q^{3}}{360}+\frac{8 \beta^{2} M^{6} Q^{3}}{140}+2 \beta M^{4} Q^{2} \gamma \\
& -\frac{48 \beta M^{6} Q^{3} \gamma}{180}
\end{aligned}
$$


Journal of Thermal Engineering, Research Article, Vol. 5, No. 1, pp. 76-92, January, 2019

$$
\begin{aligned}
& \bar{q}_{b}=\left[\begin{array}{l}
1+\beta+\frac{\beta M^{2} Q}{2}\left(x^{2}-1\right)-\frac{\beta M^{2}}{2}\left(1-x^{2}\right)+\frac{\beta M^{4} Q}{24}\left(5-6 x^{2}+x^{4}\right)+\frac{\beta^{2} M^{2} Q}{2}\left(1-x^{2}\right)- \\
\frac{\beta^{2} M^{4} Q^{2}}{24}\left(5-6 x^{2}+x^{4}\right)+\frac{\beta^{2} M^{4} Q^{2}}{30}\left(1-x^{6}\right)+\frac{\beta M^{2} Q \gamma}{2}\left(1-x^{2}\right)-\frac{\beta M^{4} Q^{2} \gamma}{24}\left(5-6 x^{2}+x^{4}\right)+ \\
{\left[\beta M^{2}-\beta M^{2} Q \gamma-\beta^{2} M Q\right]\left[\frac{M^{2}}{24}\left(5-6 x^{2}+x^{4}\right)-\frac{M^{4} Q}{720}\left(61-75 x^{2}+15 x^{4}-x^{6}\right)-\right.} \\
\frac{\beta M Q}{24}\left(5-6 x^{2}+x^{4}\right)+\frac{\beta M^{4} Q^{2}}{720}\left(61-75 x^{2}+15 x^{4}-x^{6}\right)-\frac{\beta M^{4} Q^{2}}{1680}\left(27-28 x^{2}+x^{8}\right)- \\
\left.\frac{M^{2} Q \gamma}{24}\left(5-6 x^{2}+x^{4}\right)+\frac{M^{4} Q^{2} \gamma}{720}\left(61-75 x^{2}+15 x^{4}-x^{6}\right)\right]+\frac{\beta^{2} M^{2}}{2}\left(1-x^{6}\right)+\frac{\beta^{2} M^{4} Q}{24}\left(5+6 x^{2}-x^{4}\right) \\
-\frac{\beta^{3} M^{2} Q}{2}\left(1-x^{2}\right)-\frac{\beta^{3} M^{4} Q^{2}}{24}\left(5+6 x^{2}-x^{4}\right)-\frac{\beta^{3} M^{4} Q^{2}}{30}\left(1-x^{6}\right)-\frac{\beta^{2} M^{2} Q \gamma}{2}\left(1-x^{2}\right)- \\
\frac{\beta^{2} M^{4} Q^{2} \gamma}{24}\left(5+6 x^{2}-x^{4}\right)+\frac{\beta^{2} M^{6} Q^{2}}{120}\left(11+15 x^{2}-5 x^{4}-x^{6}\right)-\frac{\beta^{3} M^{4} Q^{2}}{24}\left(5+6 x^{2}-x^{4}\right)- \\
\frac{\beta^{3} M^{6} Q^{3}}{120}\left(11-15 x^{2}+5 x^{4}-x^{6}\right)+\frac{\beta^{3} M^{6} Q^{3}}{1680}\left(13-28 x^{6}+15 x^{8}\right)+\frac{\beta^{2} M^{4} Q^{2} \gamma}{24}\left(5-6 x^{2}+x^{4}\right) \\
-\frac{\beta^{2} M^{6} Q^{3} \gamma}{120}\left(11-15 x^{2}+5 x^{4}-x^{6}\right)+\frac{\beta^{2} M^{4} Q}{6}\left(1-x^{4}\right)-\frac{\beta^{2} M^{6} Q^{2}}{180}\left(13-15 x^{4}+2 x^{6}\right)- \\
\frac{\beta^{3} M^{4} Q^{2}}{6}\left(1-x^{4}\right)+\frac{\beta^{3} M^{6} Q^{3}}{360}\left(13-15 x^{4}+2 x^{6}\right)-\frac{\beta^{3} M^{6} Q^{3}}{140}\left(1-x^{8}\right)-\frac{\beta^{2} M^{4} Q^{2} \gamma}{2}\left(1-x^{4}\right)+ \\
\frac{\beta^{2} M^{6} Q^{3} \gamma}{180}\left(13-15 x^{4}+2 x^{6}\right)
\end{array}\right] \\
& \left(M^{2} Q x+M^{2} x-\frac{M^{4}}{6}\left(3 x-x^{3}\right)-\beta M^{2} Q x+\frac{\beta M^{4} Q^{2}}{6}\left(3 x-x^{3}\right)-\frac{\beta M^{4} Q^{2} x^{5}}{6}-M^{2} Q \gamma x+\frac{M^{4} Q^{2} \gamma}{6}\left(3 x-x^{3}\right)\right. \\
& +\left(\mathrm{M}^{2}-M^{2} Q \gamma-\beta M Q\right)\left[\frac{M^{2}}{6}\left(3 x-x^{3}\right)+\frac{M^{4} Q}{120}\left(25 x-10 x^{3}+x^{5}\right)-\frac{\beta M Q}{6}\left(3 x-x^{3}\right)-\right. \\
& \left.\frac{\beta M^{4} Q^{2}}{120}\left(25 x-10 x^{3}+x^{5}\right)+\frac{\beta M^{4} Q^{2}}{210}\left(7 x-x^{7}\right)-\frac{M^{2} Q \gamma}{6}\left(3 x-x^{3}\right)-\frac{M^{4} Q^{2} \gamma}{120}\left(25 x-10 x^{3}+x^{5}\right)\right]- \\
& \beta M^{2} x+\frac{\beta M^{4} Q}{6}\left(3 x-x^{3}\right)+\beta^{2} M^{2} Q x-\frac{\beta^{2} M^{4} Q^{2}}{6}\left(3 x-x^{3}\right)+\frac{\beta^{2} M^{4} Q^{2} x^{5}}{5}+\beta M^{2} Q \gamma x- \\
& \frac{\beta M^{4} Q^{2} \gamma}{6}\left(3 x-x^{3}\right)+\frac{\beta M^{4} Q}{6}\left(3 x-x^{3}\right)-\frac{\beta M^{6} Q^{2}}{60}\left(15 x-10 x^{3}+3 x^{5}\right)-\frac{\beta^{2} M^{4} Q}{6}\left(3 x-x^{3}\right)+ \\
& \frac{\beta^{2} M^{6} Q^{3}}{60}\left(15 x-10 x^{3}+3 x^{5}\right)-\frac{\beta^{2} M^{6} Q^{3}}{70}\left(7 x^{5}-5 x^{7}\right)-\frac{\beta M^{4} Q^{2} \gamma}{6}\left(3 x-x^{3}\right)+\frac{\beta M^{6} Q^{3} \gamma}{60}\left(15 x-10 x^{3}+3 x^{5}\right) \\
& -\frac{2 \beta M^{4} Q x^{3}}{3}+\frac{\beta M^{6} Q^{2}}{15}\left(5 x^{3}-x^{5}\right)+\frac{2 \beta^{2} M^{4} Q^{2} x^{3}}{3}-\frac{\beta^{2} M^{6} Q^{3}}{30}\left(5 x^{3}-x^{5}\right)+\frac{2 \beta^{2} M^{6} Q^{3} x^{7}}{35}+ \\
& \frac{2 \beta M^{4} Q^{2} \gamma x^{3}}{3}-\frac{\beta M^{6} Q^{3} \gamma}{15}\left(5 x^{3}-x^{5}\right)
\end{aligned}
$$


Journal of Thermal Engineering, Research Article, Vol. 5, No. 1, pp. 76-92, January, 2019

Table 1. Comparison of various values of $X$ for temperature profiles. When $M=0.5, \gamma=\beta=Q=0$.

\begin{tabular}{|l|l|c|}
\hline $\mathbf{X}$ & $\begin{array}{l}\boldsymbol{\theta}(\mathbf{X}) \\
\text { Joneidi et al. [12] }\end{array}$ & $\begin{array}{c}\text { Present } \\
\text { work }\end{array}$ \\
\hline $\mathbf{0 . 0 0}$ & 0.8868188841 & 0.8880 \\
$\mathbf{0 . 0 5}$ & 0.8870960292 & 0.8883 \\
$\mathbf{0 . 1 0}$ & 0.8879276383 & 0.8891 \\
$\mathbf{0 . 1 5}$ & 0.8893142310 & 0.8905 \\
$\mathbf{0 . 2 0}$ & 0.8912566748 & 0.8924 \\
$\mathbf{0 . 2 5}$ & 0.8937561827 & 0.8949 \\
$\mathbf{0 . 3 0}$ & 0.8968143173 & 0.8979 \\
$\mathbf{0 . 3 5}$ & 0.9004329897 & 0.9015 \\
$\mathbf{0 . 4 0}$ & 0.9046144623 & 0.9056 \\
$\mathbf{0 . 4 5}$ & 0.9093613475 & 0.9103 \\
$\mathbf{0 . 5 0}$ & 0.9146766135 & 0.9155 \\
$\mathbf{0 . 5 5}$ & 0.9205635842 & 0.9213 \\
$\mathbf{0 . 6 0}$ & 0.9270259345 & 0.9277 \\
$\mathbf{0 . 6 5}$ & 0.9340677074 & 0.9347 \\
$\mathbf{0 . 7 0}$ & 0.9416933025 & 0.9422 \\
$\mathbf{0 . 7 5}$ & 0.9499074872 & 0.9504 \\
$\mathbf{0 . 8 0}$ & 0.9587153946 & 0.9591 \\
$\mathbf{0 . 8 5}$ & 0.9681225298 & 0.9684 \\
$\mathbf{0 . 9 0}$ & 0.9781347745 & 0.9783 \\
$\mathbf{0 . 9 5}$ & 0.9887583840 & 0.9889 \\
$\mathbf{1 . 0 0}$ & 1.0000000000 & 1.0000 \\
\hline
\end{tabular}

\section{RESULT AND DISCUSSIONS}

This section shows the effect of various parameters on heat transfer on the straight fin exposed to convecting environmental condition where both thermal conductivity and internal heat generation are temperature dependent. The temperature distribution across the fin, heat flux at the fins base and efficiency is reported graphically as seen in figures 2-8. The figure 2 illustrates the effect of the thermo-geometric parameter (M) on the temperature distribution across the fin length which plays an important role as it determines the amount of heat dissipated from the fin to the environment. It can be depicted that as $\mathrm{M}$ increases the temperature distribution decreases across the fins length which can be physically explained by the rapid drop in fins temperature as convective heat transfer to the environment increases.

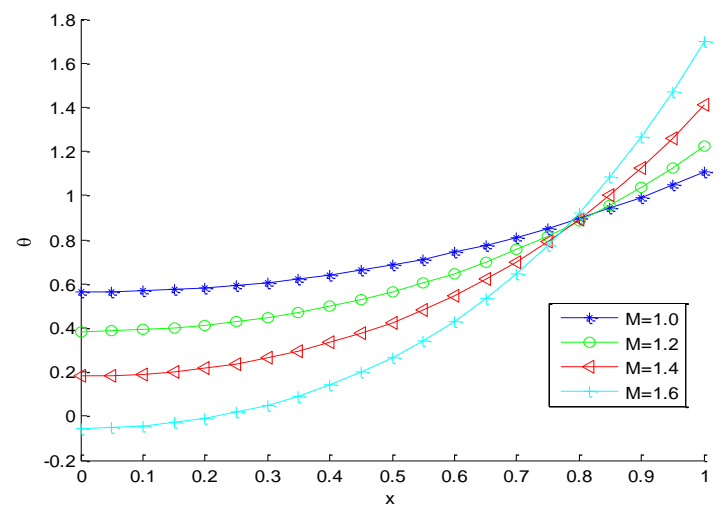

Figure 2. Effect of thermo geometric parameter on temperature distribution, where $Q=0.8, \gamma=0.4, \beta=0.8$ 
Journal of Thermal Engineering, Research Article, Vol. 5, No. 1, pp. 76-92, January, 2019

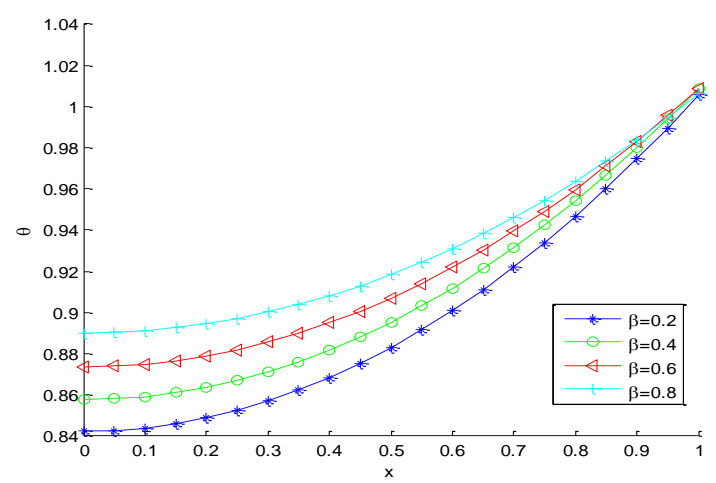

Figure 3. Effect of thermal conductivity parameter on temperature distribution, where $\mathrm{Q}=0.8, \gamma=0.4, \mathrm{M}=0.5$

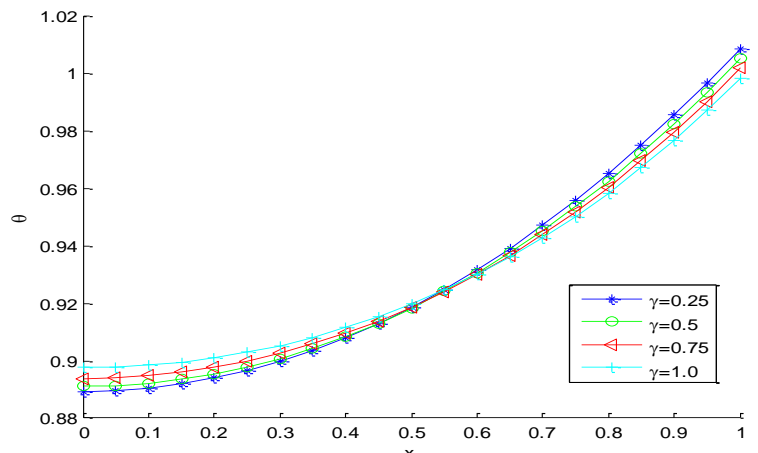

Figure 4. Effect of internal heat generation on temperature distribution, where $\mathrm{Q}=0.8, \beta=0.8, \mathrm{M}=0.5$

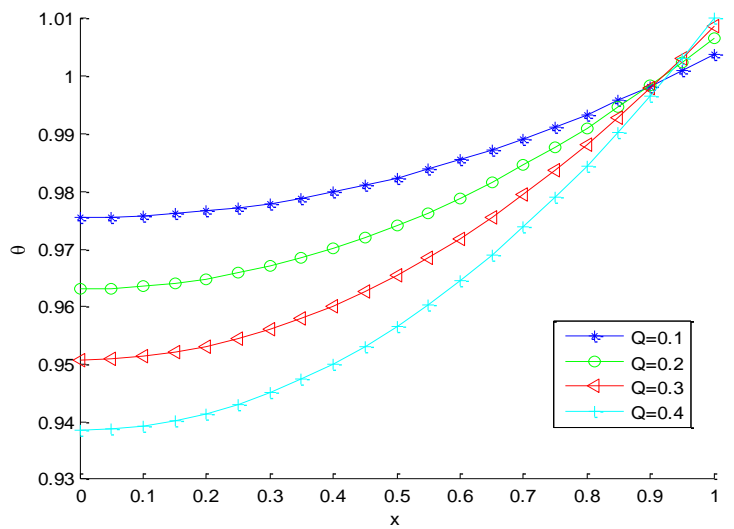

Figure 5. Effect of heat transfer coefficient on temperature distribution, where $\gamma=0.4, \beta=0.8, M=0.5$ 
Journal of Thermal Engineering, Research Article, Vol. 5, No. 1, pp. 76-92, January, 2019

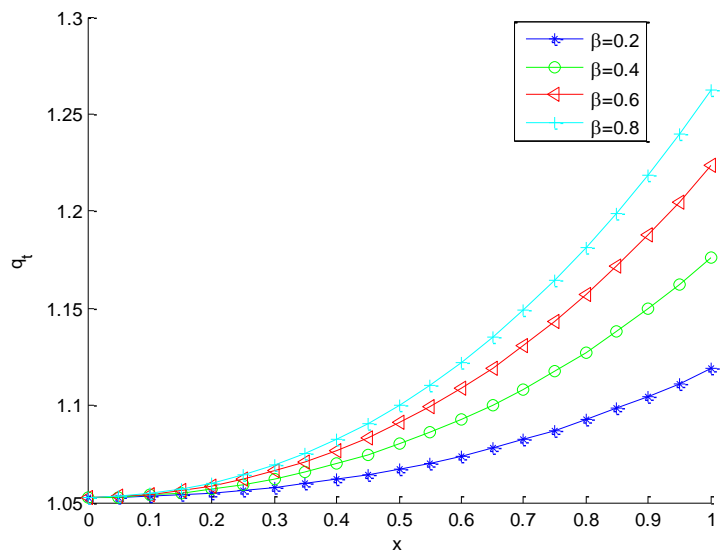

Figure 6. Effect of thermal conductivity parameter on heat flux, where $Q=0.8, \gamma=0.4, M=0.5$

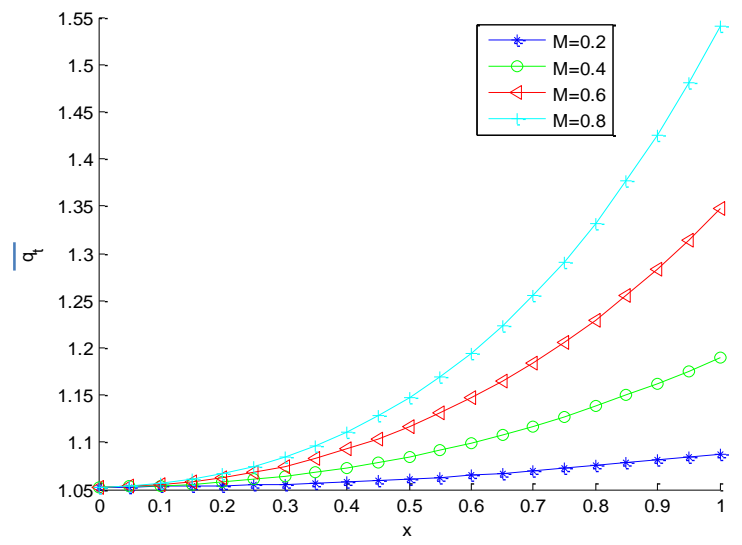

Figure 7. Effect of thermo-geometric parameter on heat flux, where $Q=0.8, \beta=0.8, \gamma=0.5$

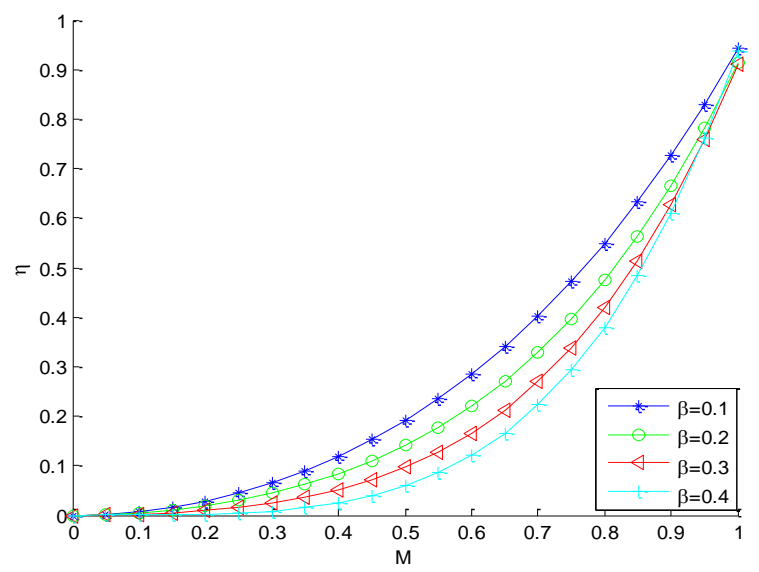

Figure 8. Effect of thermal conductivity on fins efficiency, where $Q=\gamma=1$. 
Effect of thermal conductivity $(\beta)$ on heat transfer across the fin can be seen in figure 3 . Increase in fins thermal conductivity causes an increased heat transfer across fins surface as a result of increased heat been conducted from the prime surface to the fin where it is observed in figure 3 that temperature distribution increases with corresponding increase in $\beta$. Internal heat generation $(\gamma)$ effect on fins heat transfer is illustrated in figure 4 where it is shown that temperature distribution increases near the fin base and as we move towards the fin tip temperature distribution decreases because gradient of temperature is such that heat extracted from the prime source alongside internal heat generated from the fin is expelled into the environment in such a manner heat is not stored in the fin so as to prevent heat flow back to the prime surface, which defeats the aim of fin. As heat transfer coefficient (Q) increases as observed in figure 5, temperature distribution decreases as a result of enhanced convective heat transfer due to flow caused by large temperature difference between the fin and surrounding environment.

Major analyses in fins problems include the heat flux effect on thermal performance where it is obvious that parameters such as thermal conductivity and thermo-geometric parameter have high influence on the functionality of the fins since they have direct impact on heat rate. Heat flux effect with respect to the fins base is observed in figure 6 where it is seen that with increasing thermal conductivity $(\beta)$ heat flux increase significantly at the base of the fin. In figure 7 effect of thermo geometric parameter (M) is demonstrated, where an increase in (M) causes a corresponding increase in heat flux as depicted from the plot. The fins efficiency is observed in figure 8 where it is illustrated that at increasing thermal conductivity, efficiency improves due to increasing amount of heat transferred to the environment.

\section{CONCLUSION}

In this paper, the heat transfer across convective straight fins with temperature dependent conductivity and internal heat generation is considered adopting the adomian decomposition method. The obtained approximate solution is used to study the effect of temperature dependent parameters during heat transfer on the temperature profile. Also the heat flux across the axial distance is investigated with respect to the fins base. It is observed from results that heat loss is significant at fins base towards the upper tip at increasing effect of thermo geometric parameter $(\mathrm{M})$ and thermal conductivity $(\beta)$. Though $\mathrm{M}$ parameter causes an increased heat loss compared to $\beta$. The study provides useful insight to the operational and thermal performance of fins application in heat exchange media such as radiator, gas and steam plants, boiler, refrigeration and air conditioning equipment's and oil pipe lines amongst others.

\section{NOMENCLATURE}

$\mathrm{Bi}$

$\mathrm{L}$

$\mathrm{h}$

A

$\mathrm{x}$

$Q=\frac{q_{a} A_{c}}{h_{b} p\left(T_{b}-T_{\infty}\right)}$

$q_{i} \quad$ Uniform internal heat generation, $w / \mathrm{m}^{3}$

$\overline{q_{b}}$

$X=\frac{x}{b}$

$H=\frac{h}{h_{b}}$

$\mathrm{P}$
Biot number

Length of fin, $m$

Heat transfer coefficient, $\mathrm{wm}^{-2} \mathrm{k}^{-1}$

Cross sectional area, $\mathrm{m}^{2}$

Axial distance, $\mathrm{m}$

Dimensionless heat transfer

Dimensionless heat flux

Dimensionless fin length

Dimensionless heat transfer coefficient at fin base

Perimeter of fin, $m$ 


$\begin{array}{ll}K=\frac{k}{k_{a}} & \text { Dimensionless thermal conductivity } \\ M^{2}=\frac{P h_{a} L_{2}}{A_{c} k_{a}} & \text { Dimensionless geometric fin parameter } \\ \gamma=\psi\left(T_{b}-T_{\infty}\right) & \text { Dimensionless internal heat generation Parameter } \\ \delta & \text { Fin thickness, m } \\ \beta=\lambda\left(T_{b}-T_{\infty}\right) & \text { Thermal conductivity parameter } \\ \theta=\frac{T-T_{\infty}}{T_{b}-T_{\infty}} & \text { Dimensionless temperature } \\ \mathrm{b} & \text { Fin base } \\ \infty & \text { Ambient temperature }\end{array}$

\section{REFERENCES}

[1] Atay, M.T., Coskun, S.B. (2007). Comparative analysis of power law fin type problems using variational iteration method and finite element method. Mathemathical Problem in Engineering, article ID 42072.

[2] Chowdbury, M.S.H., Hashim, I., Abdulaziz, O. (2009).Comparison of homotopy analysis and homotopy perturbation method for purely nonlinear fin type problems. Communication in Nonlinear science and Numerical simulations, 14, 371-378.

[3] Kraus, A.D., Aziz, A., Welty, J.R. (2002). Extended surface heat transfer, John wiley and sons, New York.

[4] Ghasemi, S.E., Hatami, M., Ganji, D.D. (2014). Thermal analysis of convective fins with temperature dependent thermal conductivity and heat generation. Case studies in Thermal Engineering, 4, 1-8.

[5] Hatami, M., Mehdizadeh Ahangar, G.H.R, Ganji, D.D., Boubakar, K. (2014). Refrigeration analysis for fully wet semi-spherical porous fins. Energy Conversion and Management, 84,533-540.

[6] Hatami, M., Ganji, D.D., Gorji-Bandpy, M. (2014). Numerical study of finned type heat exchangers for ICEs exhaust waste heat recovery. Case Studies in Thermal Engineering, 4, 53-64.

[7] Hatami, M., Ganji, D.D., Gorji-Bandpy, M. (2014). Experimental and thermodynamical analysis of diesel exhausts vortex generator heat exchanger for optimizing its operating condition. Applied Thermal Engineering, 30, 1-12.

[8] Hatami, M., Jafaryar, M, Ganji, D.D., Gorji-Bandpy, M. (2014). Optimization of finned tube heat exchangers for diesel exhaust waste recovery using CFD and CCD techniques. International Communication in Heat and Mass Transfer, 57, 254-263.

[9] Fernandez, A. (2009). On some approximate methods for nonlinear models. Applied Mathematical Computation, $215,168-174$.

[10] Sadris, S., Raveshi, M.R., Amiri, S. (2012).Efficiency analysis of straight fin with variable heat transfer coefficient and thermal conductivity. Journal of Mechanical Science and Techonology, 26, 1283-1290.

[11] Moradi, A., Almadikia, H. (2011).Investigation of effect of thermal conductivity on straight fin performance with DTM. International Journal of Engineering and Applied science, 1, 42-54.

[12] Joneidi, A.A., Ganji, D.D., Babaelah, M. (2009). Differential transform method to determine fin efficiency of convective straight fins with temperature dependent thermal conductivity. International Communication in Heat and Mass Transfer, 39, 757-762.

[13] Hatami, M., Ganji, D.D. (2014). Thermal behavior of longitudinal convective-radiative porous fins with different section, shapes and ceramic materials $\left(\mathrm{SiC}\right.$ and $\left.\mathrm{Si}_{3} \mathrm{~N}_{4}\right)$. Ceramic International, 40, 6765-6775.

[14] Hatami, M., Ganji, D.D. (2014). Investigation of refrigeration efficiency for fully wet circular porous fins with variable sections by combined heat and mass transfer analysis. International Journal of refrigeration, 40, 140-151. 
[15] Arslanturk, A. (2005). A decomposition method for fin efficiency of convective straight fin with temperature dependent thermal conductivity. International Communication in Heat and Mass Transfer, 32, 831-841.

[16] Aziz, A., Enamul-Huq, S.M. (1973).Perturbation solution for convecting fin with temperature dependent thermal conductivity. Journal of Heat Transfer, 97, 300-310.

[17] Aziz, A. (1977). Perturbation solution for convective fin with internal heat generation and temperature dependent thermal conductivity. International Journal of Heat and Mass Transfer, 20, 1253-1255.

[18] Mosayebidorcheh, S., Ganji, D.D., Masoud, F. (2014). Approximate solutions of nonlinear heat transfer equation of a fin with power law temperature dependent thermal conductivity and heat transfer coefficient. Propulsion and Power Research, 14, 41-47.

[19] Ganji, D.D., Dogonchi, A.S. (2014). Analytical investigation of convective heat transfer coefficient and heat generation. International Journal of Physical Science, 9, 466-474.

[20] Aziz, A., Bouaziz, M.N. (2011). A least squares method for a longitudinal fins with temperature dependent internal heat generation. Energy Conversion and Management, 52, 2876-2882.

[21] Hosseini, K., Daneshian, B., Amanifard, N., Ansari, R. (2012) .Homotopy analysis method for a fin with temperature dependent thermal conductivity and internal heat generation. International Journal of Nonlinear Science, 14, 210-210.

[22] Moitheki, R.J., Hayat, T., Malik, M.Y. (2010). Some exact solutions of the fin problem with power law temperature dependent thermal conductivity. Nonlinear Analysis for Real World Application, 11, 3287-3294.

[23] Khan, F., Ahmadzadeh, R.M., Hamedi, N.H. (2009). Analytical solutions and efficiency of nonlinear fin problem with temperature dependent thermal conductivity and heat transfer co-efficient. Communication in Nonlinear Science and Numerical Simulation, 14, 3327-3338.

[24] Domairry, G., Fazeli, M. (2009). Homotopy analysis method to determine the fin efficiency of convective straight fin with temperature dependent thermal conductivity. Communication in Nonlinear Science and Numerical Simulation, 14, 489-499.

[25] Cosun, S.B., Atay, M.T. (2008). Fin efficiency analysis of convective straight fin with temperature dependent thermal conductivity using variational iteration method. Applied Thermal Engineering, 28, 2345-2352.

[26] Languri, E.M., Ganji, D.D., Jamshidi, N. (2008). Variational iteration and homotopy perturbation methods for fin efficiency of convective straight fins with temperature dependent thermal conductivity. $5^{\text {th }}$ WSEAS International Conference on Fluid Mechanics, Acapulco, Mexico.

[27] Oguntala, G., Sobamowo G. (2016). Garlerkin method of weighted residuals for convective straight fins with temperature dependent conductivity and internal heat generation. International Journal of Engineering and Technology, 6, 432-442.

[28] Filobello-Niño, U., Vazquez-Leal, H., Boubaker, K., Khan, Y., Perez-Sesma, A., Sarmiento Reyes, A., Jimenez-Fernandez, V.M., Diaz-Sanchez, A., Herrera-May, A., Sanchez-Orea J., Pereyra-Castro, K. (2013). Perturbation Method as a Powerful Tool to Solve Highly Nonlinear Problems: The Case of Gelfand's Equation. Asian Journal of Mathematics and Statistics, DOI: 10.3923 /ajms.2013.

[29] Lim, C.W., Wu, B.S. (2002). A modified Mickens procedure for certain non-linear oscillators. Journal of Sound and Vibration, 257, 202-206.

[30] Cheung, Y.K., Chen, S.H., Lau S.L. (1991). A modified Lindsteadt-Poincare method for certain strongly nonlinear oscillators. International Journal of Non-Linear Mechanics, 26, 367-378.

[31] Lewis, R.W., Nithiarasu, P., Seatharamu, K.N. (2004). Fundamentals of the finite element method for heat and fluid flow, Antony Rowe Ltd, Wiltshire, Great Britain.

[32]Mercan, H., Atalik, K. (2018). Numerical investigation of blood flow features in intracranial saccular aneurysms. Journal of Thermal Engineering, 4 (7), 1867-1878.

[33] Tokgoz,N., Alic, E., Kaska, O., Aksoy, M.M. (2018). The numerical study of heat transfer enhancement using AL2O3-water nanofluid in corrugated duct application. Journal of Thermal Engineering, 4(3), 1984-1997.

[34] Belhadj, A., Boucherafa, R., Saim, R. (2018). A numerical study of forced convective flow in microchannel heat sinks with periodic-expansion in construction cross section. Journal of Thermal Engineering, 4(3), 1912-1925. 
Journal of Thermal Engineering, Research Article, Vol. 5, No. 1, pp. 76-92, January, 2019

[35] Kilic, M. (2018). Numerical investigation of heat transfer from porous plate with transpiration cooling. Journal of Thermal Engineering, 4(1), 1632-1647. 